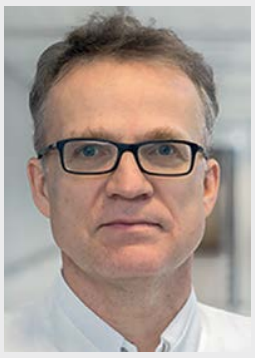

Gernot Keyßer

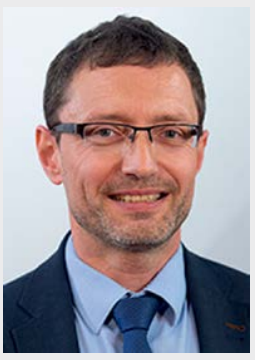

Eugen Feist

\section{Herzlich willkommen Professor Gaulke!}

Liebe Kollegen und Leser der Aktuellen Rheumatologie,

wir freuen uns außerordentlich Herrn Prof. Dr. med. Ralf Gaulke im Herausgeberteam der Aktuellen Rheumatologie zu begrüßen. Eigentlich bedarf es für seine Person keiner ausführlichen Vorstellung, da er innerhalb der internistischen und orthopädischen Rheumatologie gleichermaßen ein gut bekannter und hochgeschätzter Kollege ist. Auch in der Aktuellen Rheumatologie ist Herr Prof. Gaulke kein neues Gesicht, da er bereits seit vielen Jahren als Mitglied im Editorial Board unserer Zeitschrift sehr aktiv ist und wiederholt tolle Impulse und Ideen in die Gestaltung der Aktuellen Rheumatologie eingebracht hat.

In seiner Funktion als Leiter der Sektion „Obere Extremität, Fuß- und Rheumachirurgie“ an der Unfallchirurgischen Klinik der Medizinischen Hochschule Hannover ist Herr Prof. Gaulke tagtäglich intensiv in die orthopädische Versorgung von Patienten mit rheumatischen Erkrankungen eingebunden und wird mit seiner großen praktischen Erfahrung eine nunmehr noch wertvollere fachliche Bereicherung für unsere Aktuelle Rheumatologie sein.

Mit seiner Expertise und seiner wissenschaftlichen Ausrichtung im Bereich der rheumatologischen Orthopädie und Traumatologie ist Herrn Prof Gaulke eine idealen Ergänzung in unserem Team.

Er ist Präsident der Deutschen Gesellschaft für Orthopädische Rheumatologie (DGORh) und Vorstandsmitglied der Deutsche Gesellschaft für Orthopädie und Orthopädische Chirurgie (DGOOC) und damit ausgezeichnet mit anderen Experten seines Fachgebietes vernetzt. Gemeinsam werden wir somit auch in Zukunft sowohl internistisch- als auch orthopädisch-rheumatologische Aspekte ausgewogen und in hoher Qualität in unserer Zeitschrift präsentieren können.

Lieber Herr Prof. Gaulke, wir freuen uns sehr auf eine konstruktive Zusammenarbeit!

Eugen Feist

Gernot Keyßer 